\title{
O-III-Laborreform
}

\section{Allergologische Labordiagnostik in Gefahr!}

\author{
Wir sehen uns akut mit einer \\ bedrohlichen Entwicklung im \\ Bereich des O-III-Labors konfrontiert. \\ Hintergrund ist die seit längerem \\ kritisierte Selbstzuweisung von \\ Fällen und deren Abrechnung im \\ Allergielabor.
}

A m 26. April 2012 hat die Vertreterversammlung der Kassenärztlichen Bundesvereinigung (KBV) beschlossen, dass für alle O-III-Laborerbringer, die nicht Laborärzte sind, eine fallzahlabhängige Mengengrenze in Euro eingeführt wird. Leistungen oberhalb dieser Grenze werden nicht mehr vergütet. Vorgeschlagen sind für

- Rheumatologen, Endokrinologen $40 €$, — Nuklearmediziner, Hämatologen $21 €$, _ Dermatologen, Pneumologen, Gynäkologen, Urologen und alle übrigen Arztgruppen $4 € /$ Fall/Quartal.

Es ist eine Ausnahmeregelung im Einzelfall möglich, dies müssen die KVen vor Ort noch festlegen.

\section{Neuregelung schon vor der Tür}

Die Neuregelung soll bereits zum dritten, spätesten zum vierten Quartal 2012 in Kraft treten! Wir werden zwar mit den Laborärzten gleichgestellt, was den geplanten bundesweiten Vorwegabzug der entstandenen Kosten betrifft, es wird aber eine Quotierung - wie oben skizziert - kommen. Die Hausärzte haben vor, gegen einen generellen Vorwegabzug zu klagen. Sie wollen erreichen, dass der Vorwegabzug der Laborleistungen ausschließlich aus dem Facharzttopf genommen wird. Dies hätte aber zumindest eine Änderung des Trennungsfaktors zur Folge.
Waren die Investitionen umsonst?

Wir Allergologen haben bisher viel Geld in unser O-III-Labor investiert, wir haben RiliBÄK (Richtlinie der Bundesärztekammer zur Qualitätssicherung laboratoriumsmedizinischer Untersuchungen) und ISO-Zertifizierung mitgemacht. Wir haben unser Laborpersonal ausgebildet, Laborgeräte, Klimaanlagen und vieles mehr angeschafft. All dies soll nun ohne Beteiligung von Allergologen mit Laborberechtigung in einer plötzlichen Aktion über die Sommerzeit, in der sich die KBV wohl weniger Proteste ausrechnet, auf einen Schlag vernichtet werden. Wir werden noch nicht einmal von der KBV angehört.

\section{Therapie und Diagnostik aus einer Hand}

Das O-III-Labor in Großlaboren wird mit Diagnostika von Herstellern erstellt, die keine eigenen Therapielösungen anbieten. Dies führt bei Phytotherapeutika (und das sind Hyposensibilisierungslösungen) dazu, dass die Lösungen zum Test im Labor eine andere biologische Wirksamkeit haben als die Therapielösung. Für eine sinnvolle Theragnostik ist es aber erforderlich, dass Therapie und Diagnostik aus einer Hand erfolgen und zwar unter Aufsicht des Arztes, der die Therapie durchführt. Diese Richtigkeitskontrolle kann kein patientenferner Laborarzt leisten.
Der Bestand einer fachärztlich allergologischen Diagnostik und traditionell etablierte Strukturen werden vernichtet. Das O-III-Labor wird auf die Laborärzte übertragen, die keine Allergietests durchführen, Anamnese und Patienten nicht kennen und die keine Therapie durchführen. Das im Hintergrund drei große Laborunternehmen stehen, die keine Arztpraxen mehr sind, sondern Investmentgesellschaften, sei hier nur erwähnt. Unsere Ausbildung zum Allergologen ist keinen Cent mehr wert. Im Gegenteil, wir dürfen die Pricktestlösungen noch selbst bezahlen, da sie keinen Praxisbedarf darstellen. Wie soll Allergologie so noch in der Praxis überleben?

\section{Unterstützung erbeten!}

Der AeDA-Vorstand bittet alle Mitglieder um Unterstützung. Schreiben Sie an Ihre KV und stellen Sie ihre Problemlage dar. Erläutern Sie die Bedeutung der geplanten Regelung für Ihre Praxis und die Notwendigkeit einer adäquaten allergologischen Versorgung mit angemessener Vergütung. Fordern Sie eine Ausnahmeregelung für Ihre Praxis. Schließen Sie sich mit Ihren Berufsverbänden unserem Protest bei der KBV und Ihren lokalen KVen an. Informieren Sie die Presse und die Patientenverbände über die drohende Entwicklung.

Der AeDA-Vorstand 